\title{
A Re-Evaluation of Allometric Relationships for Circulating Concentrations of Glucose in Mammals
}

\author{
Colin G. Scanes \\ Department of Biological Science, University of Wisconsin Milwaukee, Milwaukee, WI, USA \\ Email: Scanes@uwm.edu
}

Received 10 August 2015; accepted 19 April 2016; published 22 April 2016

Copyright (C) 2016 by author and Scientific Research Publishing Inc.

This work is licensed under the Creative Commons Attribution International License (CC BY). http://creativecommons.org/licenses/by/4.0/

(c) () Open Access

\begin{abstract}
Purpose: The present study examined the putative relationship between circulating concentrations of glucose and $\log _{10}$ body weight in a large sample size $(270)$ of wild species but with domesticated animals excluded from the analyses. Methods: A data-set of plasma/serum concentration of glucose and body weight in mammalian species was developed from the literature. Allometric relationships were examined. Results: In contrast to previous reports, no overall relationship for circulating concentrations of glucose was observed across 270 species of mammals (for $\log _{10}$ glucose concentration adjusted $R^{2}=\mathbf{- 0 . 0 0 3}$; for glucose concentration adjusted $R^{2}=\mathbf{- 0 . 0 0 3}$ ). In contrast, a strong allometric relationship was observed for circulating concentrations of glucose in Primates (for $\log _{10}$ glucose concentration adjusted $R^{2}=0.511$; for glucose concentration adjusted $R^{2}=0.480$ ). Conclusion: The absence of an allometric relationship for circulating concentrations of glucose was unexpected. A strong allometric relationship was seen in Primates.
\end{abstract}

\section{Keywords}

Glucose, Allometric, Mammals, Primates

\section{Introduction}

Glucose in the blood is the principal energy source for brain functioning and but glucose can be used as the energy source for multiple other tissues. A relationship between metabolic rate and circulating concentration of glucose has been reported [1] with higher blood concentration of glucose with increasing metabolic rates in across vertebrates. Moreover, there is a negative relationship between blood concentrations of glucose and body weight (log) in mammals (blood: [2]; serum: [3]). In birds, an allometric relationship for circulating concentra- 
tions of glucose was reported in one study (blood: [4]) but not in another (plasma/serum: [5]). This leads us to question whether an allometric relation for circulating concentrations of glucose, in fact, exists in mammals. The presence of such a relationship would be consistent with the relationship between basal metabolic rate being proportional to either the body weight to the power two thirds (2/3) (reviewed: [6]) or three quarters (3/4) [7] [8].

What is not clear is whether the supposed relationship between circulating concentrations of glucose and log body weight is real reflecting a true allometric relationship (and reduced needs for energy in larger animals) or represents an artifact of species included in previous analyses and the inclusion of domesticated animals in the analyses. Domesticated animals have been selected for growth and larger body size and consequently lower circulating concentrations of glucose [9]. There are also marked phylogenic differences in circulating concentrations of glucose within the Class Mammalia [10]. The present study re-examines the relationship between circulating concentrations of glucose and body weight in mammals with a large sample size (270) of wild species but with domesticated animals excluded from the analyses.

\section{Materials and Methods}

\subsection{Databases}

A database was assembled for serum/plasma concentrations of glucose in wild species of mammals using the published or calculated mean for the species based on rigorous and systematic series of searches of the literature [10] together with body weights principally from the Animal Diversity Web. This is presented in Table 1.

Table 1. Database of plasma/serum concentrations of glucose (from [11]) and log body weight).

\begin{tabular}{|c|c|c|c|}
\hline & \multicolumn{3}{|c|}{ Species } \\
\hline & & Glucose (mM) & Log B.Wt. \\
\hline Platypus & Ornithorhynchus anatinus & 4.3 & 3.217 \\
\hline Short-beaked Echida & Tachyglossus aculeatus & 4.2 & 3.653 \\
\hline Western Quoll & Dasyurus geoffroii & 6.2 & 3.041 \\
\hline American Woolly Opossum & Caluromys derbianus & 5 & 2.489 \\
\hline Grayshort-tailed Opossum & Monodelphis domestica & 5.3 & 2.088 \\
\hline Common Opossum & Didelphis marsupialis & 5.1 & 3.185 \\
\hline Virginia Opossum & Didelphis virginiana & 4.8 & 3.597 \\
\hline Common Wallaroo & Macropus robustus & 4.8 & 4.477 \\
\hline Red Kangaroo & Macropus rufus & 4.7 & 4.954 \\
\hline Tammar Wallaby & Macropus eugenii & 5.2 & 3.878 \\
\hline Brush-tailed Rock-Wallaby & Petrogale penicillata & 6.2 & 3.872 \\
\hline Quokka & Setonix brachyurus & 4.3 & 3.538 \\
\hline Tasmanian Pademelon & Thylogale billardierii & 4.5 & 3.86 \\
\hline Common Brush-tail Possum & Trichosurus vulpecula & 7.62 & 3.455 \\
\hline Mountain Brushtail Possum & Trichosurus cunninghami & 6.8 & 3.544 \\
\hline Gilbert's Potoroo & Potorous gilbertii & 9.1 & 3.296 \\
\hline Sugar Glider & Petaurus breviceps & 4.2 & 2.041 \\
\hline Northern Hairy-nosed Wombat & Lasiorhinus krefftii & 8 & 4.512 \\
\hline Southern Hairy-nosed Wombat & Lasiorhinus latifrons & 6.2 & 4.407 \\
\hline Lesser Hedgehog tenrec & Echinops telfairi & 4.4 & 2.301 \\
\hline Rock Hyrax & Procavia capensis & 3.7 & 3.58 \\
\hline African Elephant & Loxadonta africana & 4.6 & 6.638 \\
\hline Asian Elephant & Elephas maximus & 5.55 & 6.602 \\
\hline West Indian Manatee & Trichechus manatus & 4.55 & 5.681 \\
\hline Aardvark & Orycteropus afer & 5.1 & 4.785 \\
\hline
\end{tabular}




\section{Continued}

Nine-banded armadillo

Three-Banded armadillo

Gilbert's Potoroo

Sugar Glider

Northern Hairy-nosed Wombat

Southern Hairy-nosed Wombat

Lesser Hedgehog tenrec

Rock Hyrax

African Elephant

Asian Elephant

West Indian Manatee

Aardvark

Nine-banded armadillo

Three-Banded armadillo

Brown-throated Three-toed Sloth

Hoffmann's Two-Toed Sloths

Southern Two -toed Sloth

Giant Anteater

European Hedgehog

Four-toed Hedgehog

South African Hedgehog

Japanese Shrew Mole

Least Shrew

Old World Mole

Pallas’s Mastiff Bat

Great Fruit-eating Bat

Common Vampire Bat

Malaysian Flying-fox

Rodriguez Island Flying-fox

Egyptian Fruit-bat

Wahlberg's Epauletted Fruit-bat

Small Flying-fox

Red Panda

Striped Skunk

Raccoon

Kinkajou

Ringtail

South American Coati

White-nosed Coati

Eurasian otter

North American river otter

Sea Otter

Giant Otter

\section{Dasypus novemcinctus \\ Tolypeutes matacus \\ Potorous gilbertii \\ Petaurus breviceps \\ Lasiorhinus krefftii \\ Lasiorhinus latifrons \\ Echinops telfairi \\ Procavia capensis \\ Loxadonta africana \\ Elephas maximus \\ Trichechus manatus \\ Orycteropus afer \\ Dasypus novemcinctus \\ Tolypeutes matacus \\ Bradypus variegatus \\ Choloepus hoffmanni \\ Choloepus didactylus \\ Myrmecophaga tridactyla \\ Erinaceous europaeus \\ Atelerix albiventris \\ Atelerix frontalis \\ Urotrichus talpoide \\ Cryptotis parva \\ Talpa europaea}

Molossus molossus

Artibeus lituratus

Desmodus rotundus

Pteropus vampyrus

Pteropus rodricensis

Rousettus aegyptiacus

Epomophorus wahlbergi

Pteropus hypomelanus

Ailurus fulgens

Mephitis mephitis

Procyon lotor

Potos flavus

Bassariscus astutus

Nasua nasua

Nasua narica

Lutra lutra

Lontra Canadensis

Enhydra lutris

Pteronura brasiliensis
3.752

3.176

3.296

2.041

4.512

4.407

2.301

3.58

6.638

6.602

5.681

4.785

3.752

3.176

3.6375

3.778

3.778

4.548

3

2.778

2.547

1.083

0.653

2

1.079

1.813

1.477

3.929

2.512

2.097

1.903

2.717

3.695

3.544

3.7853

3.5185

3.0338

3.6532

3.6021

3.8293

3.9778

4.4698

4.3802

3.677 


\section{Continued}

American Mink
European Badger
European Polecat
Walrus
Australian Sea Lion
California Seal Lion
Northern Fur Seal
Harbor Seal
Harp Seal
Hooded Seal

Northern Elephant Seal

American Black Bear

Brown Bear

Malayan Sun Bear

Giant Panda

Sloth Bear

Spectacled Bear

Polar Bear

Binturong

Canada Lynx

Eurasian Lynx

European Wildcat

Sand Cat

Bobcat

Cougar

Iriomote Cat

Jaguar

Tiger

Cheetah

Egyptian Mongoose

Slender-tailed Meerkat

Dwarf mongoose

Aardwolf

Spotted Hyena

Striped Hyena

Hunting Dog

Grey Wolf

Coyote

Golden Jackal

Maned Wolf

Crab-eating Fox

Ranch Gray Fox

Raccoon Dog

\begin{tabular}{|c|c|c|}
\hline Mustela vison & 6.9 & 3.061 \\
\hline Meles meles & 6.1 & 4.066 \\
\hline Mustela putorius & 9.3 & 2.9811 \\
\hline Odobenus rosmarus & 5.4 & 6.0212 \\
\hline Neophoca cinerea & 4.3 & 5.3064 \\
\hline Zalophus californianus & 7.5 & 5.2844 \\
\hline Callorhinus ursinus & 5.8 & 5.23 \\
\hline Phoca vitulina & 10.5 & 5.061 \\
\hline Phoca groenlandica & 9.8 & 5.105 \\
\hline Cystophora cristata & 7.1 & 5.362 \\
\hline Mirounga angustirostris & 5.8 & 6.161 \\
\hline Ursus americanus & 4 & 5.35 \\
\hline Ursus arctos & 6.65 & 5.531 \\
\hline Helarctos malayanus & 5.2 & 4.663 \\
\hline Ailuropoda melanoleuca & 5.1 & 5.011 \\
\hline Melursus Ursinus & 5.1 & 4.989 \\
\hline Tremarctos Ornatus & 5.7 & 5.114 \\
\hline Ursus aritimus & 6.2 & 5.677 \\
\hline Arctictis binturong & 7.1 & 4.161 \\
\hline Lynx canadensis & 7.6 & 4.037 \\
\hline Lynx lynx & 7.4 & 4.431 \\
\hline Felis silvestris & 9.3 & 3.628 \\
\hline Felis margarita & 8.2 & 3.38 \\
\hline Felis rufus & 7.2 & 3.978 \\
\hline Felis concolor & 8.35 & 4.872 \\
\hline Felis iriomotensis & 9.1 & 3.568 \\
\hline Panthera onca & 4.6 & 5.009 \\
\hline Panthera tigris & 7.1 & 5.41 \\
\hline Acinonyx jubatus & 6.5 & 4.667 \\
\hline Herpestes ichneumon & 9.7 & 3.458 \\
\hline Suricata suricatta & 6.8 & 2.86 \\
\hline Helogale parvula & 7.6 & 2.439 \\
\hline Proteles cristata & 6 & 4.041 \\
\hline Crocuta crocuta & 7.2 & 4.796 \\
\hline Hyaena hyaena & 6.1 & 4.544 \\
\hline Lycaon pictus & 6.8 & 4.431 \\
\hline Canis lupus & 5.6 & 4.712 \\
\hline Canis latrans & 8.2 & 4.14 \\
\hline Canis aureus & 6.6 & 3.954 \\
\hline Chrysocyon brachyurus & 5.9 & 2.86 \\
\hline Cerdocyon thous & 13.7 & 2.439 \\
\hline Urocyon cinereoargenteus & 7.6 & 4.041 \\
\hline Nyctereutes procyonoides & 3.3 & 3.845 \\
\hline Vulpes macrotis & 7.2 & 3.342 \\
\hline
\end{tabular}




\section{Continued}

Swift Fox

Red Fox

Fossa

African Wild Ass

Grevy's Zebra

Mountain Zebra

Ongar

Przewalski horse

Plains Zebra

Black Rhinoceros

Indian Rhinoceros

Sumatran Rhinoceros

White Rhinoceros

Baird's Tapir

Brazilian Tapir

Malayan Tapir

Mountain Tapir

Barking Deer

Eld's Deer

Elk

Red Deer

Fallow Deer

Chital deer

Moose

White-tailed Deer

Père David's Deer

$$
\text { Pudú }
$$

Caribou

Roe Deer

Rusa Deer

Sambar Deer

Pronghorn

Impala

Cuvier's Gazelle

Dama Gazelle

Dorcas Gazelle

Goitered Gazelle

Grant's Gazelle

Springbok

Brindled Wildebeest

Black Wildebeest

Bontebok

Bongo

ommon Eland

Vulpes velox

Vulpes fulva

Cryptoprocta ferox

Equus africanus

Equus grevyi

Equus zebra

Equus hemionus

Equus przewalski

Equus quagga

Diceros bicornis

Rhinoceros unicornis

Dicerorhinus sumatrensis

Ceratotherium simum

Tapirus bairdii

Tapirus terrestris

Tapirus indicus

Tapirus pinchaque

Muntiacus muntjak

Panolia eldii

Cervus canadensis

Cervus elaphus

Dama dama

Axis axis

Alces alces

Odocoileus virginianus

Elaphus davidianus

Pudu pudu

Rangifer tarandus

Capreolus capreolus

Cervus timorensis

Cervus unicolor

Antilocapra americana

Aepyceros melampus

Gazella cuvieri

Gazella dama

Gazella dorcas

Gazella subgutturosa

Gazella granti

Antidorcas marsupialis

Connochaetes taurinus

Connochaetes gnou

Damaliscus dorcas

Tragelaphus eurycerus
3.398

4.045

4.796

4.544

4.431

4.712

4.14

3.954

5.439

6.041

6.243

6.146

6.401

5.352

5.301

5.597

5.201

4.407

5

5.45

5.204

4.74

4.72

5.716

4.987

5.271

5

5.271

4.414

5.061

5.234

4.767

4.72

4.544

4.74

4.243

4.481

4.746

4.597

5.288

5.161

4.829

5.488

5.813 


\section{Continued}

Nilgai
Greater Kudu
Water Buffalo
American Bison
European Bison
Bushbuck
Lesser Kudu
Barbary Sheep
Spanish Ibex
Southern Chamois

Rocky Mountain Bighorn Sheep

Stone Sheep

Mouflon

Maxwell's Duiker

Adax Antelope

Gemsbok Oryx

Scimitar-horned Oryx

Roan Antelope

Sable Antelope

Waterbuck

Lechwe

Mountain Reedbuck

Mrs Gray’s Waterbuck

Guanaco

Giraffe

Okapi

Hippopotamus

Pygmy Hippopotamus

Buru babirusa

Red River Hog

Warthog

Wild Boar

Collared Peccary

Chacoan Peccary

Bryde's Whale

Bowhead Whale

Dall Porpoise

Finless Porpoise

Gray Whale

Amazon River Dolphin

Commerson's Dolphin

Bottlenose Dolphin

Common Dolphin

False Killer Whale
Boselaphus tragocamelus

Tragelaphus strepsiceros

Bubalus bubalis

Bison bison

Bison bonasus

Tragelaphus scriptus

Ammelaphus imberbis

Ammotragus lervia

Capra pyrenaica

Rupicapra pyrenaica

Ovis Canadensis

Ovis dalli

Ovis orientalis

Philantomba maxwellii

Addax nasomaclatus

Oryx gazella

Oryx dammah

Hippotragus equinus

Hippotragus niger

Kobus ellipsiprymnus

Kobus leche

Redunca fulvorufula

Kobus megaceros

Lama guanicoe

Giraffa camelopardalis

Okapia johnstoni

Hippopotamus amphibius

Choeropsis liberiensis

Babyrousa babyrussa

Potamochoerus porcus

Phacochoerus africanus

$$
\text { Sus scrofa }
$$

Tayassu tajacu

Catagonus wagneri

Balaenoptera edeni

Balaena mysticetus

Phocoenoides dalli

Neophocaena phocaenoides

Eschrzchtzus robustus

Inia geoffrensis

Cephalorhynchus commersonii

Tursiops truncatus

Delphinus delphius

Pseudorca crassidens
5.25

5.337

5.628

5.787

5.954

4.721

4.916

5.021

4.76

4.568

4.954

4.9

4.628

3.778

4.996

5.322

5.301

5.419

5.36

5.362

4.977

4.477

4.954

5.061

6.192

5.398

6.462

4.337

4.854

4.944

5

5.228

4.301

4.591

7.162

7.9

5.243

4.845

7.161

5.151

4.699

5.58

5.072

6.482 


\section{Continued}

\begin{tabular}{|c|c|c|c|}
\hline Killer Whale & Orcinus orca & 8.95 & 6.857 \\
\hline Pacific White-sided Dolphin & Lagenorhynchus obliquidens & 6.65 & 5.013 \\
\hline Pilot Whale & Globicephala macrorhynchus & 8.1 & 6.342 \\
\hline Spotted Dolphin & Stenella attenuata & 7.7 & 4.954 \\
\hline Beluga whale & Delphinapterus leucas & 6.4 & 6.154 \\
\hline Brazilian Porcupine & Coendou prehensilis & 5.5 & 3.7 \\
\hline Black-tailed Hairy Dwarf Porcupine & Coendou melanurus & 3.95 & 3.279 \\
\hline Coypu & Myocastor coypus & 8.9 & 3.875 \\
\hline Greater Cane Rat & Thryonomys swinderianus & 5.15 & 3.374 \\
\hline Dusky-footed Wood Rat & Neotoma fuscipes & 6.4 & 2.366 \\
\hline Meadow Vole & Microtus pennsylvanicus & 5.7 & 1.69 \\
\hline Pine Vole & Microtus pinetorum & 5.6 & 1.407 \\
\hline Norwegian Lemming & Lemmus lemmus & 7.3 & 1.875 \\
\hline Muskrat & Ondatra zibethicus & 13.4 & 2.945 \\
\hline Central Rock-rat & Zyzomys pedunculatus & 3.8 & 1.978 \\
\hline House Mouse & Mus musculus domesticus & 5.05 & 1.322 \\
\hline Libyan Jird & Meriones libycus & 6.4 & 1.929 \\
\hline Persian Jird & Meriones persicus & 6.7 & 2.061 \\
\hline Short-tailed Bandicoot Rat & Nesokia indica & 6.7 & 2.456 \\
\hline Indian Gerbil & Tatera indica & 5.9 & 2 \\
\hline Norway Rat & Rattus norvegicus & 6.3 & 2.505 \\
\hline Sand Rat & Psammomys obesus & 4.3 & 1.574 \\
\hline Kangaroo Rat & Dipodomys merriami & 14.3 & 1.653 \\
\hline Gray squirrel & Sciurus carolinensis & 7.7 & 2.736 \\
\hline Thirteen-lined ground squirrel & Spermophilus tridecemlineatus & 9 & 2.097 \\
\hline White-Tailed Prairie Dog & Cynomys leucurus & 8 & 3.075 \\
\hline Guinea Pig & Cavia porcellus & 6.7 & 2.954 \\
\hline Long-tailed Chinchilla & Chinchilla lanigera & 7 & 2.813 \\
\hline Tuco-tuco & Ctenomys talarum & 5.2 & 2.146 \\
\hline Bank Vole & Myodes glareolus & 3.95 & 1.267 \\
\hline Deer mice & Peromyscus maniculatus & 5.6 & 1.23 \\
\hline Golden or Syrian Hamster & Mesocricetus auratus & 5.8 & 2.051 \\
\hline Northern red-backed Vole & Myodes rutilus & 4.4 & 1.477 \\
\hline Fat-tailed Jird & Pachyuromys duprasi & 12.2 & 1.562 \\
\hline Gerbil & Meriones unguiculatus & 4.3 & 1.966 \\
\hline Libyan jird & Meriones libycus & 6.4 & 1.906 \\
\hline Short-tailed Bandicoot Rat & Nesokia indica & 6.7 & 2.458 \\
\hline Spiny mice & Acomys cahirinus & 8.8 & 1.699 \\
\hline Blind Subterranean Mole Rat & Spalax judaei & 8.61 & 2.511 \\
\hline Black-tailed Jackrabbit & Lepus californicus & 8.7 & 3.312 \\
\hline European Brown Hare & Lepus europaeus & 12.9 & 3.677 \\
\hline Eastern Cottontail Rabbit & Sylvilagus floridanus & 12.9 & 3.066 \\
\hline Riparian Brush Rabbit & Sylvilagus bachmani & 6.3 & 2.845 \\
\hline Black Lemur & Eulemur macaco & 4.9 & 3.254 \\
\hline
\end{tabular}




\section{Continued}

\begin{tabular}{|c|c|c|c|}
\hline Black-and-white ruffed Lemur & Varecia variegata & 6.7 & 3.544 \\
\hline Eastern Lesser Bamboo Lemur & Hapalemur griseus & 6.4 & 2.85 \\
\hline White-Fronted Brown Lemur & Eulemur fulvus & 3 & 3.447 \\
\hline Red ruffed Lemur & Varecia rubra & 5.5 & 3.641 \\
\hline Ring-tailed Lemur & Lemur catta & 7.5 & 3.362 \\
\hline Verreaux’s Sifaka & Propithecus verreauxi Strepsirhini & 6.6 & 3.58 \\
\hline Bushbaby & Galago crassicaudatus Strepsirhini & 5.4 & 3.176 \\
\hline Slender Loris & Loris tardigradus & 7.5 & 2.279 \\
\hline Three-striped Night Monkey & Aotus trivirgatus & 9.1 & 3.086 \\
\hline Cotton-Top Tamarin & Saguinus oedipus & 14.7 & 2.505 \\
\hline Common Marmoset & Callithrix jacchus & 10.6 & 2.491 \\
\hline White-Footed Tamarin & Saguinus leucopus & 9.4 & 2.699 \\
\hline Goeldi’s Monkey & Callimico goeldii & 8.9 & 2.563 \\
\hline Tufted Capuchin & Cebus apella & 7.2 & 3.491 \\
\hline Common Squirrel Monkey & Saimiri sciureus & 5.53 & 2.82 \\
\hline De Brazza’s Monkey & Cercopithecus neglectus & 4.3 & 3.76 \\
\hline Hamadryas Baboon & Papio hamadryas & 4.3 & 4.365 \\
\hline Lion-tailed Macaque & Macaca silenus & 5.8 & 3.771 \\
\hline Bonobo & Pan paniscus & 4.1 & 4.592 \\
\hline Chimpanzee & Pan troglodytes & 4.6 & 4.61 \\
\hline Gorilla & Gorilla gorilla & 3.81 & 5.13 \\
\hline Orangutan & Pongo pygmaeus & 4.3 & 4.94 \\
\hline Rhesus Monkey & Macaca mulatta & 3.83 & 3.813 \\
\hline Stump-tailed Macaque & Macaca arctoides & 4.3 & 3.947 \\
\hline Three-striped Night Monkey & Aotus trivirgatus & 9.1 & 3.097 \\
\hline Brown Woolly Monkey & Lagothrix lagotricha & 5.9 & 3.845 \\
\hline Owl Monkey & Aotus nancymaae & 6.5 & 3.097 \\
\hline Red howler Monkey & Alouatta seniculus & 5.7 & 3.785 \\
\hline Black-capped or Bolivian squirrel monkey & Saimiri boliviensis & 7.8 & 2.888 \\
\hline Central American squirrel or Colombian monkey & Saimiri oerstedii & 6.4 & 2.888 \\
\hline Tonkean Macaque & Macaca tonkeana & 3.4 & 4.099 \\
\hline Sooty Mangabey & Cercocebus atys & 4.8 & 3.895 \\
\hline Northern Plains Gray Langur & Semnopithecus entellus & 5.9 & 4.14 \\
\hline Chacma or Cape baboon & Papio ursinus & 5.4 & 4.362 \\
\hline Celebes black Macaque & Macaca nigra & 4 & 4.061 \\
\hline Crab-eating or Cynomolgus Macaque & Macaca fascicularis & 5.89 & 3.699 \\
\hline Grivet or African Green monkey & Chlorocebus aethiops & 5.87 & 3.602 \\
\hline
\end{tabular}

\subsection{Statistics}

Allometric relationships (comparing both serum/plasma concentrations of glucose and $\log _{10}$ serum/plasma concentrations of glucose with $\log _{10}$ body weight) across the Class Mammalia were analyzed by linear regression (Microsoft Excel). The data were also analysed separately for major groups of mammals including Marsupial mammals, Eutherian mammals, for taxa within the Eutherian mammals, namely Glires, Euarchonta, Laurasiatheria, Afrotheria and Xenarthra (following the relationships advanced in [11]-[13]) together with Orders and sub-orders within the Laurasiatheria and Euarchonta where there is sufficient data for analysis. 


\section{Results}

There was not a relationship between circulating concentrations of glucose and $\log _{10}$ body weight in wild mammals (circulating concentrations of glucose: adjusted $\mathrm{R}^{2}=-0.003 ; \log _{10}$ circulating concentrations of glucose: adjusted $\mathrm{R}^{2}=-0.003$ ) (Table 2 and Table 3) (Figure 1 ). Similarly, there was no allometric relationships when data for marsupial or eutherian mammals were analyzed separately (Table 2 and Table 3) or with major taxa within the Eutheria.

However, there was a strong relationship between circulating concentrations of glucose and $\log _{10}$ body weight in some taxa; specifically across species within the Order Primates (circulating concentrations of glucose: adjusted $\mathrm{R}^{2}=0.480 ; \mathrm{p}<0.001$; $\log _{10}$ circulating concentrations of glucose: adjusted $\left.\mathrm{R}^{2}=0.511 \mathrm{p}<0.001\right)$ (Table 2 and Table 3). Moreover there were moderate allometric relationships in the Orders Perissodactyla and Carnivora (Adjusted $\mathrm{R}^{2}=0.085$; $\mathrm{p}<0.05$ ) (Table 2 and Table 3). Within the Order Primates, there was an even stronger allometric relationship in the Sub-order Haplorhini (New World and Old World monkeys together with Apes) (circulating concentrations of glucose: adjusted $\mathrm{R}^{2}=0.597 ; \mathrm{p}<0.001$; $\log _{10}$ circulating concentrations of glucose: adjusted $\mathrm{R}^{2}=0.657$; $\mathrm{p}<0.001$ ) (Figure 2) but no allometric relationship in in the Sub-order Strepsirhini. Within the Order Carnivora, the moderate allometric relationship was no longer significant when the data were examined within Sub-orders (circulating concentrations of glucose: Sub-order Caniformia-adjusted $\mathrm{R}^{2}=$ 0.059, $\mathrm{p}=0.064$; Feliformia-adjusted $\mathrm{R}^{2}=0.098, \mathrm{p}=0.128$ ). Despite there being data on circulating concentrations of glucose in a large sample size (76 species) within the Order Cetartiodactyla, there was no allometric relationship observed. Moreover, if the data for Cetacean species was analyzed separately, again no allometric relationship was observed (circulating concentrations of glucose: Cetacea-adjusted $\mathrm{R}^{2}=-0.077, \mathrm{p}=0.948$; Artiodactylia-adjusted $\mathrm{R}^{2}=0.0007, \mathrm{p}=0.312$ ).

\section{Discussion}

There was no relationship between circulating concentrations of glucose and log body weight in wild species of

Table 2. Relationship between serum/plasma concentrations of glucose and log body weight in mammalian species.

\begin{tabular}{|c|c|c|c|}
\hline Taxa & Adjusted $\mathrm{R}^{2}=(\mathrm{n}=)$ & $\mathrm{p}=$ & Slope \\
\hline Class Mammalia & $-0.003(270)$ & 0.815 & \\
\hline Infra-class Marsupialia & $-0.047(18)$ & 0.629 & \\
\hline Infra-class Eutheria (Placentalia) & $-0.004(250)$ & 0.936 & \\
\hline \multicolumn{4}{|l|}{ Within Infra-class Eutheria (Placentalia) } \\
\hline Super-order Afrotheria & $0.183(5)$ & 0.219 & \\
\hline Super-order Euarchonta & $0.480(38)$ & $8.62 \mathrm{E}-07$ & -2.33 \\
\hline Super-order Glires & $0.049(38)$ & 0.096 & \\
\hline Super-order Laurasiatheria & $-0.006(163)$ & 0.878 & \\
\hline Super-order Xenoarthra & $-0.245(6)$ & 0.906 & \\
\hline \multicolumn{4}{|l|}{ Within Laurasiatheria } \\
\hline Order Carnivora & $0.081(59)$ & 0.016 & -0.74 \\
\hline Order Cetartiodactyla & $-0.013(76)$ & 0.871 & \\
\hline Order Chiroptera & $0.209(8)$ & 0.209 & \\
\hline Order Perissodactyla & $0.235(14)$ & 0.045 & -1.12 \\
\hline \multicolumn{4}{|l|}{ Within Super-order Glires } \\
\hline Order Rodentia & $-0.018(34)$ & 0.532 & \\
\hline \multicolumn{4}{|l|}{ Within Super-order Euarchonta } \\
\hline Primates & $0.480(38)$ & $8.62 \mathrm{E}-07$ & -2.33 \\
\hline \multicolumn{4}{|l|}{ Within Primates } \\
\hline Sub-order Strepsirhini & $0.0075(9)$ & 0.337 & \\
\hline Sub-order Haplorhini & $0.597(29)$ & $5.56 \mathrm{E}-07$ & -2.69 \\
\hline
\end{tabular}


Table 3. Relationship between $\log _{10}$ serum/plasma concentrations of glucose and $\log _{10}$ body weight across mammals and in mammalian taxa.

\begin{tabular}{|c|c|c|c|}
\hline Taxa & Adjusted $\mathrm{R}^{2}=(\mathrm{n}=)$ & $\mathrm{p}=$ & Slope \\
\hline Class Mammalia & $-0.003(269)$ & 0.686 & \\
\hline Infra-class Marsupialia & $-0.044(17)$ & 0.560 & \\
\hline Infra-class Eutheria (Placentalia) & $-0.004(251)$ & 0.684 & \\
\hline \multicolumn{4}{|c|}{ Within Infra-class Eutheria (Placentalia) } \\
\hline Super-order Afrotheria & $0.179(6)$ & 0.222 & \\
\hline Super-order Euarchonta & $0.511(38)$ & $2.82 \mathrm{E}-07$ & -0.153 \\
\hline Super-order Glires & $0.068(38)$ & 0.062 & \\
\hline Super-order Laurasiatheria & $-0.006(163)$ & 0.786 & \\
\hline Super-order Xenoarthra & $-0.249(6)$ & 0.948 & \\
\hline \multicolumn{4}{|l|}{ Within Laurasiatheria } \\
\hline Order Carnivora & $0.068(59)$ & 0.0256 & -0.040 \\
\hline Order Cetartiodactyla & $-0.013(76)$ & 0.910 & \\
\hline Order Chiroptera & $0.204(8)$ & 0.146 & \\
\hline Order Perissodactyla & $0.242(14)$ & 0.0425 & -0.079 \\
\hline \multicolumn{4}{|l|}{ Within Super-order Glires } \\
\hline Order Rodentia & $-0.005(34)$ & 0368 & \\
\hline \multicolumn{4}{|l|}{ Within Super-order Euarchonta } \\
\hline Primates & $0.511(38)$ & $2.82 \mathrm{E}-07$ & -0.153 \\
\hline \multicolumn{4}{|l|}{ Within Primates } \\
\hline Sub-order Strepsirhini & $0.022(9)$ & 0.392 & \\
\hline Sub-order Haplorhini & $0.657(29)$ & $5.95 \mathrm{E}-08$ & -0.172 \\
\hline
\end{tabular}
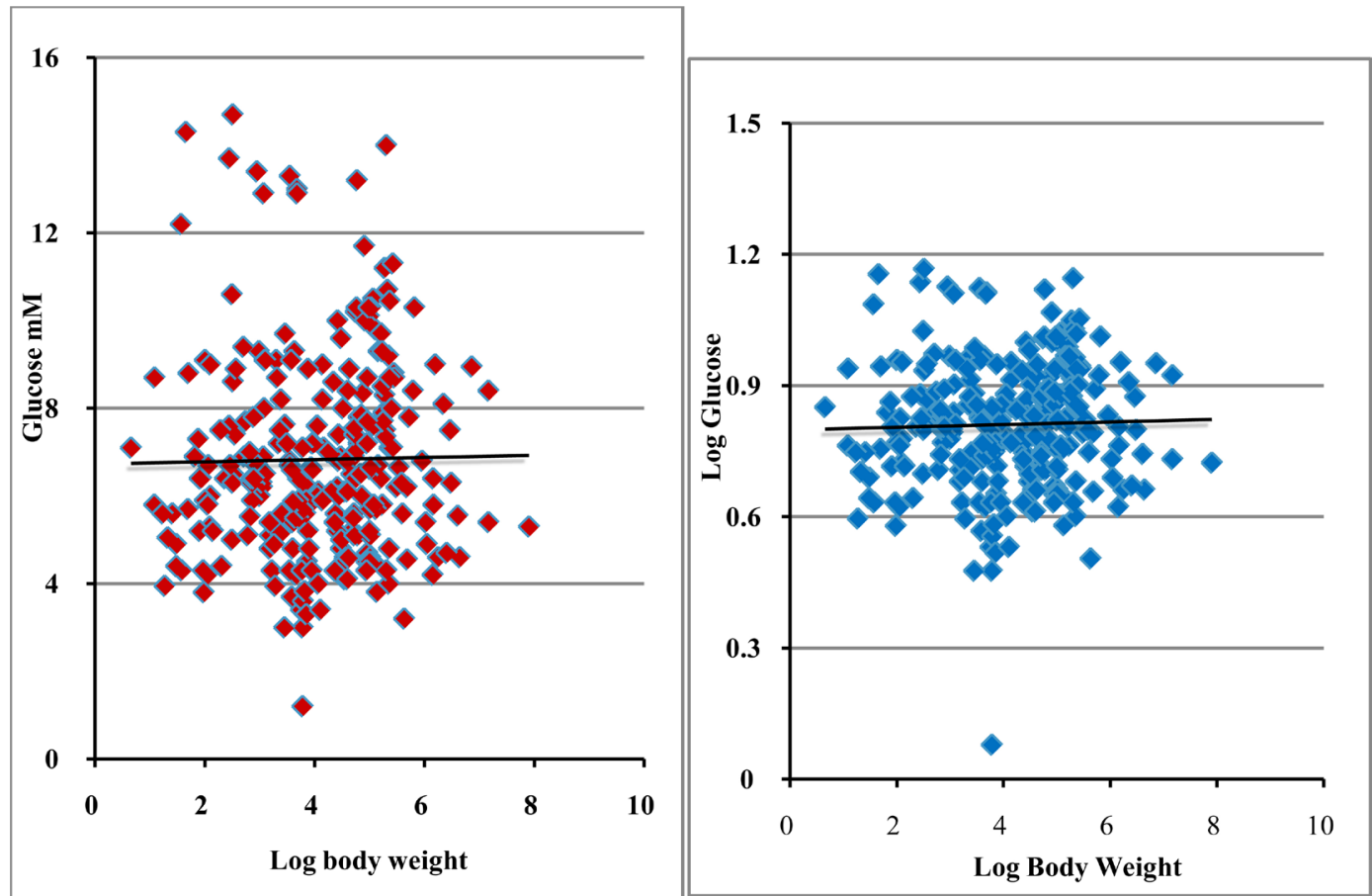

Figure 1. Relationship between plasma/serum concentration and body weight across mammalian species. Left: plas$\mathrm{ma}$ /serum concentration $(\mathrm{mM})$ and $\log _{10}$ body weight across mammalian species; Right: $\log _{10}$ plasma/serum concentration and $\log _{10}$ body weight across mammalian species (mM). 


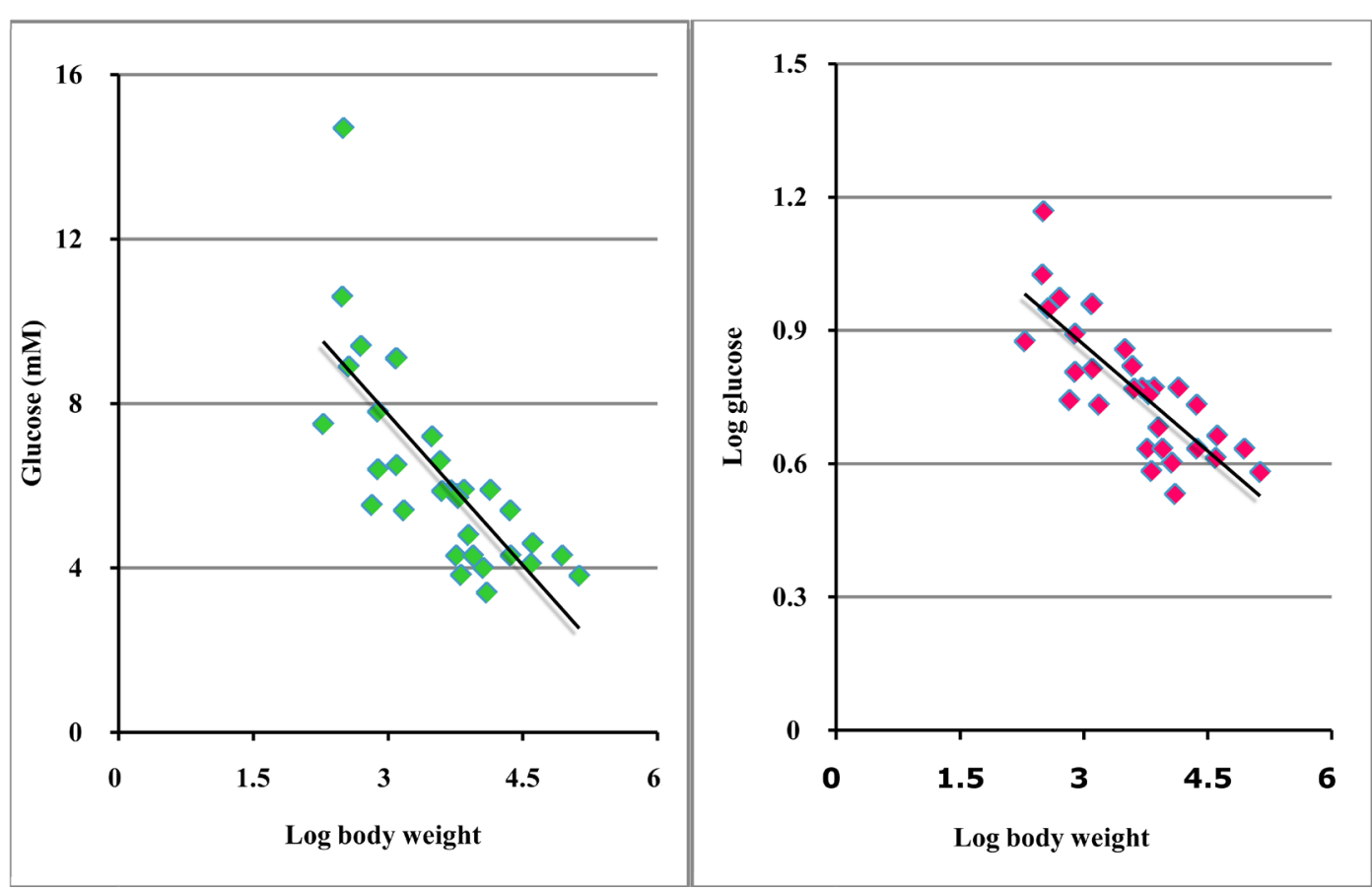

Figure 2. Allometric relationship for plasma/serum concentrations of glucose in Primate species (left glucose, right log glucose concentration).

the Class Mammalia or Infra-classes Eutheria or (Placentalia) (Table 2 and Table 3; Figure 1). This is in contrast to previous studies in mammals [2] [3]. The basis for the differences is not clear. The present study employed a much large number of species and deliberately omitted domesticated species. There were similarly no allometric relationships observed across species for many mammalian taxa including marsupials, eutherian mammals, Super-orders Afrotheria (e.g. aardvarks, elephants, sea cows), Glires (rodents, rabbits and hares), Laurasiatheria (e.g. carnivores, large herbivores and whales) and Xenoarthra (anteaters, armadillos, sloths) and in Laurasiatherian orders such as Chiroptera (bats) and Cetartiodactyla (even toed ungulates such as deer together with whales and dolphins).

There was a very strong allometric relationship between circulating concentrations of glucose and log body weight in species of the Class Primates (apes, monkeys and lemurs) (Table 2 and Table 3, Figure 2) and, particularly, in the Sub-order Haplorhini (New World and Old World monkeys together with the Apes). Body weight accounted for much of the variation in circulating concentrations of glucose in species in the Sub-order Haplorhini) (>60\%) (Figure 2). Moreover, there was an allometric relationship with circulating concentrations in species in the Orders Perissodactyla (odd toed ungulates such as horses, rhinoceroses and tapirs) and Carnivora (Table 1). It is suggested that in these taxa that the relationship between circulating concentrations of glucose and body weight is consistent to the reductions in energy requirements per unit weight with increasing body weight. Despite the strong relationship between here was insufficient data on basal metabolic rate or brain weight in primates (or within the Haplorhini) [14] for an analysis with sufficient power for significance. Alternatively, circulating concentrations of glucose may play a critical role in determining optimal body weight.

The overall conclusions are that there is no allometric relationship between circulating concentrations of glucose (or $\log _{10}$ circulating concentrations of glucose) and $\log _{10}$ body weight across species of wild mammals. However, there was a strong allometric relationship in primates, particularly in the Haplorhini (monkeys and apes).

\section{Conclusion}

No relationship was observed between circulating concentrations of glucose and $\log _{10}$ body weight in a large sample size (270) of wild species but with domesticated animals excluded from the analyses. The absence of an allometric relationship for circulating concentrations of glucose was unexpected. A strong allometric relation- 
ship was seen in Primates.

\section{Acknowledgements}

The helpful discussions with colleagues are gratefully acknowledged.

\section{References}

[1] Umminger, B.L. (1977) Relation of Whole Blood Sugar Concentrations in Vertebrates to Standard Metabolic Rate. Comparative Biochemistry and Physiology A, 56, 457-460. http://dx.doi.org/10.1016/0300-9629(77)90267-5

[2] Umminger, B.L. (1975) Body Size and Whole Blood Sugar Concentrations in Mammals. Comparative Biochemistry and Physiology A, 53, 455-458. http://dx.doi.org/10.1016/S0300-9629(75)80065-X

[3] Kjeld, M. and Ólafsson, Ö. (2008) Allometric (Scaling) of Blood Components in Mammals: Connection with Economy of Energy. Canadian Journal of Zoology 86, 890-899. http://dx.doi.org/10.1139/Z08-061

[4] Braun, E.J. and Sweazea, K.L. (2008) Glucose Regulation in Birds. Comparative Biochemistry and Physiology B, 151, 1-9. http://dx.doi.org/10.1016/j.cbpb.2008.05.007

[5] Beuchat, C.A. and Chong, C.R. (1998) Hyperglycemia in Hummingbirds and Its Consequences for Hemoglobin Glycation. Comparative Biochemistry and Physiology A, 120, 409-416. http://dx.doi.org/10.1016/S1095-6433(98)10039-9

[6] White, C.R. and Seymour, R.S. (2005) Allometric Scaling of Mammalian Metabolism. Journal of Experimental Biology, 208, 1611-1619. http://dx.doi.org/10.1242/jeb.01501

[7] Glazier, D.S. (2005) Beyond the "3/4-Power Law": Variation in the Intra- and Interspecific Scaling of Metabolic Rate in Animals. Biological reviews of the Cambridge Philosophical Society, 80, 611-662. http://dx.doi.org/10.1017/S1464793105006834

[8] Glazier, D.S. (2010) A Unifying Explanation for Diverse Metabolic Scaling in Animals and Plants. Biological Reviews of the Cambridge Philosophical Society, 85, 111-138. http://dx.doi.org/10.1111/j.1469-185X.2009.00095.x

[9] Scanes, C.G. (2014) Comparison of Circulating Concentrations of Glucose in Vertebrate and Invertebrate Taxa: Evolutionary and Physiological Implications. Trends in Comparative Biochemistry \& Physiology, 18, 15-60.

[10] Scanes, C.G. (2014) Shifts in Circulating Concentrations of Glucose in Domesticated Mammals: Is There a Consistent Adaptation to Domestication? Food and Nutrition Science, 15, 1652-1659. http://dx.doi.org/10.4236/fns.2014.517178

[11] Murphy, W.J., Eizirik, E., Johnson, W.E., Zhang, Y.P., Ryder, O.A. and O’Brien, S.J. (2001) Molecular Phylogenetics and the Origins of Placental Mammals. Nature, 409, 614-618. http://dx.doi.org/10.1038/35054550

[12] Huchon, D., Madsen, O., Sibbald, M.J., Ament, K., Stanhope, M.J., Catzeflis, F., de Jong, W.W. and Douzery, E.J. (2002) Rodent Phylogeny and a Timescale for the Evolution of Glires: Evidence from an Extensive Taxon Sampling Using Three Nuclear Genes. Molecular Biology and Evolution, 19, 1053-1065. http://dx.doi.org/10.1093/oxfordjournals.molbev.a004164

[13] Picone, B., Masters, J., Silvestro, D., Sineo, L. and DelPero, M.A. (2011) Phylogenetic Analysis of Human Syntenies Revealed by Chromosome Painting in Euarchontoglires Orders. The Journal of Mammalian Evolution, 18, 131-146. http://dx.doi.org/10.1007/s10914-010-9150-x

[14] Leonard, W.R., Robertson, M.L., Snodgrass, J.J. and Kuzawa, C.W. (2003) Metabolic Correlates of Hominid Brain Evolution. Comparative Biochemistry and Physiology A, 136, 5-15. http://dx.doi.org/10.1016/S1095-6433(03)00132-6 